\title{
Effects of iron on DNA release and biofilm development by Pseudomonas aeruginosa
}

Correspondence

Tim Tolker-Nielsen

ttn@biocentrum.dtu.dk

Received 4 December 2006

Revised 11 January 2007

Accepted 16 January 2007

\author{
Liang Yang, Kim B. Barken, Mette E. Skindersoe, Allan B. Christensen, \\ Michael Givskov and Tim Tolker-Nielsen
} Centre for BioScience and Technology, BioCentrum-DTU, Technical University of Denmark,
DK-2800 Lyngby, Denmark

\begin{abstract}
Extracellular DNA is one of the major matrix components in Pseudomonas aeruginosa biofilms. It functions as an intercellular connector and plays a role in stabilization of the biofilms. Evidence that DNA release in $P$. aeruginosa PAO1 biofilms is controlled by the las-rh/ and pqs quorum-sensing systems has been previously presented. This paper provides evidence that DNA release in $P$. aeruginosa PAO1 biofilms is also under iron regulation. Experiments involving cultivation of $P$. aeruginosa in microtitre trays suggested that pqs expression, DNA release and biofilm formation were favoured in media with low iron concentrations ( $5 \mu \mathrm{M} \mathrm{FeCl}_{3}$ ), and decreased with increasing iron concentrations. Experiments involving cultivation of $P$. aeruginosa in a flow-chamber system suggested that a high level of iron $\left(100 \mu \mathrm{M} \mathrm{FeCl}_{3}\right)$ in the medium suppressed DNA release, structural biofilm development, and the development of subpopulations with increased tolerance toward antimicrobial compounds. Experiments with $P$. aeruginosa strains harbouring fluorescent reporters suggested that expression of the pqs operon was induced in particular subpopulations of the biofilm cells under low-iron conditions $\left(1 \mu \mathrm{M} \mathrm{FeCl}_{3}\right.$ ), but repressed in the biofilm cells under high-iron conditions $(100 \mu \mathrm{M} \mathrm{FeCl})_{3}$.
\end{abstract}

\section{INTRODUCTION}

Biofilms are sessile populations of micro-organisms that are surrounded by the slime they produce, and may be attached to an inert or living surface. Biofilm formation is described as a developmental sequence that varies to some extent between bacterial species but often results in the formation of mature microbial communities with tower- or mushroom-shaped microcolonies (Davey \& O'Toole, 2000; Sauer et al., 2002; Klausen et al., 2003a). The bacteria in biofilms in medical settings often display increased resistance toward host immune responses and enhanced tolerance toward antibiotic treatment (Costerton et al., 1999). Knowledge about the molecular mechanisms that are involved in biofilm development, and the factors that lead to the formation of resistant subpopulations, may be useful for creating strategies to control biofilm formation and eradicate persistent infections.

One of the most distinctive features of biofilms is the extracellular polymeric substances that surround the bacteria and constitute the biofilm matrix. The composition of biofilm matrices varies depending upon the bacterial species and the environmental conditions, but in general they contain components such as polysaccharides, proteins

Abbreviations: $\mathrm{AHL}, \mathrm{N}$-acylhomoserine lactone; CLSM, confocal laser scanning microscope/microscopy; DDAO, 7-hydroxy-9H-(1,3-dichloro9,9-dimethylacridin-2-one; POS, Pseudomonas quinolone signal. and extracellular DNA (Sutherland, 2001; Whitchurch et al., 2002).

Evidence for a role of extracellular DNA as matrix component in Pseudomonas aeruginosa biofilms has been presented both for the $P$. aeruginosa PAO1 reference strain and for clinical $P$. aeruginosa isolates (Whitchurch et al., 2002; Nemoto et al., 2003). P. aeruginosa PAO1 biofilm development in the wells of microtitre plates was attenuated by the presence of DNase I, and biofilm formation by $P$. aeruginosa $\mathrm{PAO} 1$ in flow-chambers was almost absent when the flow-chambers were irrigated with medium containing DNase I (Whitchurch et al., 2002). In addition, young $P$. aeruginosa PAO1 biofilms that had been grown in flowchambers irrigated with DNase-free medium dispersed rapidly after addition of DNase I to the flowing medium, whereas mature $P$. aeruginosa PAO1 biofilms were not dispersed by DNase I treatment, suggesting that components other than extracellular DNA stabilize mature $P$. aeruginosa PAO1 biofilms (Whitchurch et al., 2002). Matsukawa \& Greenberg (2004) investigated the composition of the extracellular matrix of mature $P$. aeruginosa PAO1 biofilms and found that extracellular DNA was by far the most abundant polymer, although exopolysaccharide appeared to be the most critical structural matrix component. In contrast to the finding that extracellular DNA may not be the primary cell-to-cell interconnecting compound in mature $P$. aeruginosa $\mathrm{PAO} 1$ biofilms, Nemoto 
et al. (2003) found that mature biofilms formed by four different clinical $P$. aeruginosa isolates could be dispersed by DNase treatment, suggesting that extracellular DNA is the critical matrix component in mature biofilms formed by these $P$. aeruginosa strains.

Quorum sensing, a mechanism by which bacteria can monitor their cell population density through the extracellular accumulation of signalling molecules, has been shown to play a role in structural development and stabilization of $P$. aeruginosa biofilms (Davies et al., 1998). $P$. aeruginosa employs the three interconnected quorumsensing systems las, rhl and pqs to control expression of a wide range of virulence factors (Passador et al., 1993; Latifi et al., 1995, 1996; Pesci et al., 1999). The major signal molecules involved are 3-oxo-C12-homoserine lactone (3oxo-C12-HSL) in the case of the las system, C4-HSL in the case of the $r h l$ system, and 2-heptyl-3-hydroxy-4-quinolone, designated the Pseudomonas quinolone signal (PQS), in the case of the pqs system (Latifi et al., 1995, 1996; Pesci et al., 1999). Synthesis of PQS depends on several putative enzymes encoded by the pqsABCDE, pqsL, phnAB and $p q s H$ genes, as well as on a LysR-type regulator encoded by the $p q s R$ gene (also known as $m v f R$ ) (Cao et al., 2001; Gallagher et al., 2002; D'Argenio et al., 2002). Evidence has been presented that the quorum-sensing systems play a role in the formation of extracellular DNA in P. aeruginosa biofilms (Allesen-Holm et al., 2006). Quorum-sensingregulated DNA release from $P$. aeruginosa populations presumably involves lysis of a subpopulation of the cells (or lysis of DNA-containing vesicles), as experiments with lacZcontaining $P$. aeruginosa strains indicated that the formation of extracellular $\beta$-galactosidase correlated with the formation of large amounts of extracellular DNA (Allesen-Holm et al., 2006).

P. aeruginosa colonizes the lungs of cystic fibrosis (CF) patients and is a major cause of lung deterioration, health decline and death of these patients (Høiby et al., 2001). Several studies indicate that $P$. aeruginosa forms biofilms in the CF lung (e.g. Lam et al., 1980; Baltimore et al., 1989; Worlitzsch et al., 2002; Høiby et al., 2001), and the biofilm mode of growth is considered the major reason that these bacteria can not be eradicated by host defences or antibiotic treatment (Costerton et al., 1999; Høiby et al., 2001). The conditions that infecting bacteria encounter in the CF lung is of great interest, and artificial CF sputum media have been developed to enable experiments to be carried out under relevant and controllable conditions (Sriramulu et al., 2005; Palmer et al., 2005). P. aeruginosa cells in the CF lung are believed to be in intense competition for iron with the host (Haas et al., 1991; Ratledge \& Dover, 2000). Low iron concentrations were shown to promote biofilm formation in artificial CF sputum medium (Sriramulu et al., 2005). In addition to low iron levels, the presence of extracellular DNA was shown to be important for $P$. aeruginosa biofilm formation in artificial sputum medium (Sriramulu et al., 2005).
Knowledge about the factors that affect DNA release from $P$. aeruginosa is important for understanding the environmental cues that can lead to biofilm formation and increased pathogenicity of this organism. In this paper we present evidence that the level of iron in the medium affects $P$. aeruginosa DNA release and biofilm formation. The iron level was shown to have an effect on the amount of biofilm formed in the wells of microtitre trays, as well as on structural biofilm development in flow chambers, and on the development of subpopulations with increased tolerance toward antimicrobial compounds. Evidence is presented that iron affects $P$. aeruginosa DNA release and biofilm development by affecting the PQS signalling pathway.

\section{METHODS}

Bacteria and growth conditions. $P$. aeruginosa PAO1 was obtained from the Genetic Stock Center (strain PAO0001). The lasI rhlI derivative was constructed by allelic displacement in PAO1 as described by Hentzer et al. (2003). The $p q s A$, $p q s R$ and $p q s L$ mutants were constructed by D'Argenio et al. (2002) via transposon insertion in PAO1. The $g f p$-tagged PAO1 strain was constructed by insertion of a mini-Tn7-eGFP-Gm ${ }^{\mathrm{r}}$ cassette as described by Klausen et al. (2003b). Escherichia coli strains MT102 and MC1000 were used for standard DNA manipulations. LB medium (Bertani, 1951) was used to cultivate E. coli strains. Batch cultivation of $P$. aeruginosa was carried out at $30^{\circ} \mathrm{C}$ in $\mathrm{AB}$ minimal medium (Clark \& Maaløe, 1967) supplemented with $30 \mathrm{mg}$ glucose $1^{-1}$. P. aeruginosa biofilms were cultivated at $30^{\circ} \mathrm{C}$ in flow-chambers irrigated with $\mathrm{FAB}$ medium (Heydorn et al., 2000) supplemented with $0.3 \mathrm{mM}$ glucose. $\mathrm{FeCl}_{3}$ was added to the media to give the final ferric iron concentrations described in the text. Selective media were supplemented with ampicillin (Ap; $100 \mathrm{mg} \mathrm{l}^{-1}$ ), gentamicin ( $\mathrm{Gm} ; 60 \mathrm{mg} \mathrm{l}^{-1}$ ), kanamycin $\left(\mathrm{Km} ; 100 \mathrm{mg} \mathrm{l}^{-1}\right)$, streptomycin (Sm; $\left.100 \mathrm{mg} \mathrm{l}^{-1}\right)$, or tetracycline (Tc; $\left.20 \mathrm{mg}^{-1}\right)$. AB medium supplemented with $0.05 \mathrm{mg}$ propidium iodide $\mathrm{ml}^{-1}$ was used in the microtitre trays for quantification of extracellular DNA. DDAO [7-hydroxy-9H-(1,3-dichloro-9,9dimethylacridin-2-one] was used at a concentration of $1 \mu \mathrm{M}$ to stain extracellular DNA in flow-chamber-grown biofilms. Propidium iodide was used at a concentration of $0.01 \mathrm{mg} \mathrm{ml}^{-1}$ to stain dead cells in flow-chamber-grown biofilms. Syto62 was used at a concentration of $1 \mu \mathrm{M}$ to stain bacteria in flow-chamber-grown biofilms.

Measurements of pvdD expression. A classical $\beta$-galactosidase assay, described by Miller (1972), was used to measure expression of the $p v d D-l a c Z$ fusion in $P$. aeruginosa $\mathrm{PAO} 1$ cells transformed with the low-copy-number plasmid pPM190::pvdD (Rombel et al., 1995), which carries the $p v d D$ promoter fused to the E. coli lacZ gene.

Construction of the pqsA::gfp(ASV) reporter. The promoterprobe plasmid pAC37, containing a $P_{p q s A}:: g f p(A S V)$ transcriptional fusion, was constructed using standard techniques for DNA manipulations (Sambrook et al., 1989). A P. aeruginosa DNA region containing the $P_{p q s A}$ promoter was PCR amplified by use of the primers PA0996F (5'-GGAATTCTGATCAATGCCGTCGCCCCCTTGGA-3') and PA0996R (5'-GGGTACCCATGACAGAACGTTCCCTCTT-3'). A $P$. aeruginosa DNA region containing the $P_{r h l R}$ promoter and the rhlR gene was PCR amplified by use of the primers PA3477F ( $5^{\prime}$ CGTGATCAAACGGCTGGTCCGGCTCG-3') and PA3477R (5'GGAATTCAAAAAGGCCATCCGTCAGGATGGCCTTCTAGATTATCTACTCAGATGAGACCCAGCGCC-3'). An E. coli T2 transcrip-

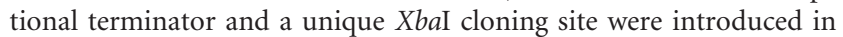
the PCR fragment through use of the PA3477R primer set. pJBA100, consisting of a NotI fragment with the $g f p(A S V)$ gene from pJBA89 
(Andersen et al., 2001) cloned in the shuttle vector pUCP22NotI, was used as scaffold. pJBA100 was digested with KpnI and EcoRI; the PCR product containing the $P_{p q s A}$ promoter was digested with $B c l \mathrm{I}$ and KpnI; and the PCR product containing the $r h l R$ gene was digested with $B c l$ and EcoRI. The expression monitor pAC36 was constructed by ligating the three DNA fragments in one reaction. Plasmid pAC37 was constructed by deleting the $r h l R$ gene on pAC36, via digestion with $X b a \mathrm{I}$ and $B c l$, Klenow treatment, and religation. The pAC37 plasmid was electroporated into $P$. aeruginosa PAO1 and the P. aeruginosa lasI rhlI mutant.

Measurement of biofilm and extracellular DNA in microtitre trays. The microtitre tray biofilm formation assay was performed essentially as described by O'Toole \& Kolter (1998). Overnight cultures were grown in $\mathrm{AB}$ medium and diluted to $\mathrm{OD}_{600} 0.001$ with fresh $\mathrm{AB}$ medium. The diluted cultures were transferred to the wells of polystyrene microtitre trays $(150 \mu \mathrm{l}$ per well $)$ and incubated for $24 \mathrm{~h}$ at $30^{\circ} \mathrm{C}$. The medium was removed from the wells and they were washed twice with $0.9 \% \mathrm{NaCl}$, stained with $0.1 \%$ crystal violet and washed twice with $0.9 \% \mathrm{NaCl}$; the crystal violet-stained biofilms were then resuspended in $96 \%$ ethanol, and biofilm cell-associated dye was measured as $A_{590}$. Measurements of extracellular DNA in $P$. aeruginosa microtitre tray cultures was done as described by AllesenHolm et al. (2006). Microtitre tray cultures were started by diluting $P$. aeruginosa overnight cultures to $\mathrm{OD}_{600} 0.001$ in $\mathrm{AB}$-glucose medium supplemented with $0.05 \mathrm{mM}$ propidium iodide and $\mathrm{FeCl}_{3}$ (as indicated) in wells of polystyrene microtitre plates $(150 \mu \mathrm{l}$ per well). The cultures were incubated for $24 \mathrm{~h}$ at $37^{\circ} \mathrm{C}$, after which propidium iodide absorbance was measured at $480 \mathrm{~nm}$ and cell density was measured at $600 \mathrm{~nm}$ by the use of a Wallac microplate reader.

DNase I treatment of biofilms in microtitre trays. Twentyfour-hour-old microtitre-tray-grown biofilms of PAO1 were washed twice with $0.9 \% \mathrm{NaCl}$ and treated with $0.9 \% \mathrm{NaCl}$ containing $100 \mu \mathrm{g}$ DNase $\mathrm{I} \mathrm{ml}^{-1}$ for $3 \mathrm{~h}$, after which the amount of biofilm was measured as described above.

Cultivation of biofilms in flow-chambers. Biofilms were grown in flow-chambers with individual channel dimensions of $1 \times 4 \times$ $40 \mathrm{~mm}$. The flow system was assembled and prepared as described previously (Sternberg \& Tolker-Nielsen, 2005). The flow-chambers were inoculated by injecting $350 \mu$ lovernight culture diluted to an $\mathrm{OD}_{600}$ of 0.001 into each flow channel using a small syringe. After inoculation, the flow channels were left without flow for $1 \mathrm{~h}$, after which medium flow was started using a Watson Marlow 205S peristaltic pump. The mean flow velocity in the flow-chambers was $0.2 \mathrm{~mm} \mathrm{~s}^{-1}$, corresponding to laminar flow with a Reynolds number of 0.02 .

Microscopy and image acquisition. All microscopy observations and image acquisitions were done with a Zeiss LSM510 confocal laser scanning microscope (CLSM) equipped with detectors and filter sets for monitoring of Gfp, propidium iodide Syto62 and DDAO fluorescence. Images were obtained using a $63 \times / 1.4$ objective or a $40 \times / 1.3$ objective. Simulated three-dimensional images and sections were generated using the IMARIS software package (Bitplane $A G)$.

Assessment of extracellular DNA in flow-chamber-grown biofilms. DDAO was added to the medium to a final concentration of $1 \mu \mathrm{M}$ to fluorescently stain extracellular DNA in flow-chambergrown biofilms. DDAO fluorescence in different biofilms was recorded with fixed settings of the CLSM.

Antimicrobial treatment. Biofilm tolerance to EDTA or ciprofloxacin was assessed by irrigating 4-day-old flow-chamber-grown $P$. aeruginosa biofilms with medium containing $50 \mathrm{mM}$ EDTA or $50 \mu \mathrm{g}$ ciprofloxacin $\mathrm{ml}^{-1}$ for $24 \mathrm{~h}$, followed by staining of the dead cells with propidium iodide, and CLSM image acquisition. To quantify survival rates, the bacteria from flow-chamber biofilms were recovered after EDTA treatment, ciprofloxacin treatment, or no antimicrobial treatment, by pumping bead-containing (Sigma glass-beads $212-300 \mu \mathrm{m})$ medium rapidly in and out of the flow-chambers using syringes. The bead-containing bacterial suspensions were vortex mixed, and the bacteria were plated on LB plates; c.f.u. were determined and survival rates calculated.

COMSTAT image analysis. CLSM images were analysed by use of the computer program COMSTAT (Heydorn et al., 2000). A fixed threshold value and connected volume filtration were used for all image stacks.

\section{RESULTS}

We initially performed experiments to determine the iron status of $P$. aeruginosa in our AB-glucose medium supplemented with different concentrations of iron. Expression of the $p v d D$ gene in $P$. aeruginosa is known to be suppressed by high levels of iron (Ochsner et al., 2002). We measured $\beta$ galactosidase activity in microtitre tray cultures of $P$. aeruginosa harbouring the low-copy-number plasmid pPM190::pvdD, which carries the $p v d D$ promoter fused to the E. coli lacZ gene (Rombel et al., 1995). The $\beta$ galactosidase activity (Miller units) was 7757, 4944, 2773 and 2206 (three replicates, SD 581, 458, 347 and 58) in cultures supplemented with 5, 10, 50 and $100 \mu \mathrm{M} \mathrm{FeCl}$. Accordingly, the selected range of iron concentrations in our medium evidently affected the iron status of the $P$. aeruginosa cells.

When $P$. aeruginosa $\mathrm{PAO} 1$ was cultivated in microtitre trays, a high cell density was reached in wells containing ABglucose medium supplemented with 100 or $50 \mu \mathrm{M}$ iron, whereas a slightly lower cell density was reached in wells containing $\mathrm{AB}$-glucose medium supplemented with $10 \mu \mathrm{M}$ iron, and a significantly lower cell density was reached in wells containing $\mathrm{AB}$-glucose medium supplemented with $5 \mu \mathrm{M}$ iron (Fig. 1A). However, the lowest iron concentration used did not limit growth dramatically, and the highest iron concentration used did not inhibit growth.

\section{Iron affects $P$. aeruginosa DNA release and biofilm formation in microtitre trays}

High concentrations of iron salts were reported to inhibit $P$. aeruginosa biofilm formation (Musk et al., 2005). Because extracellular DNA is a major matrix component of $P$. aeruginosa biofilms (Whitchurch et al., 2002; Nemoto et al., 2003; Matsukawa \& Greenberg, 2004; Allesen-Holm et al., 2006), we found it of interest to investigate whether the presence of high levels of iron might inhibit the generation of extracellular DNA from $P$. aeruginosa. In accordance with the work of Musk et al. (2005), we found that biofilm formation by $P$. aeruginosa $\mathrm{PAO} 1$ in microtitre trays decreased with increasing iron concentrations (Fig. 1B). In order to quantify the extracellular DNA generated in the microtitre tray cultures (from planktonic as well as from biofilm cells), we supplemented the medium with propidium iodide (which fluoresces when it is bound to DNA, 
(A)

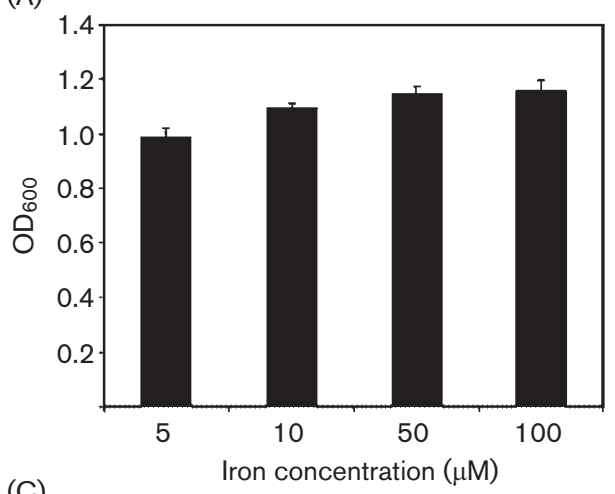

(C)

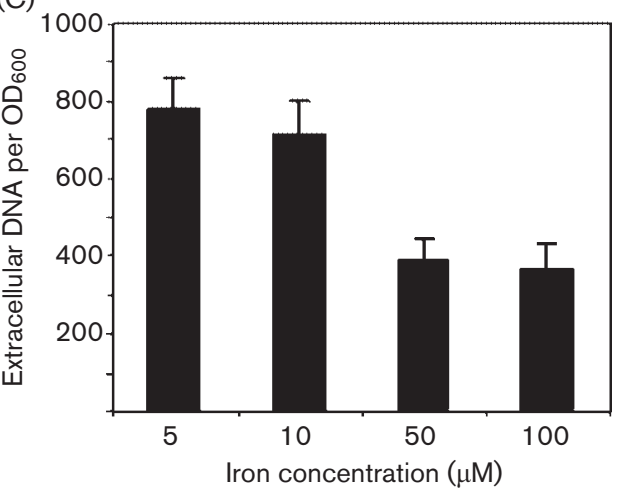

(B)

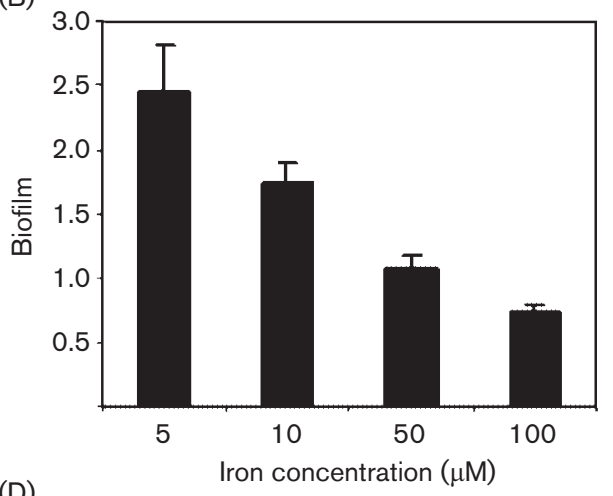

(D)

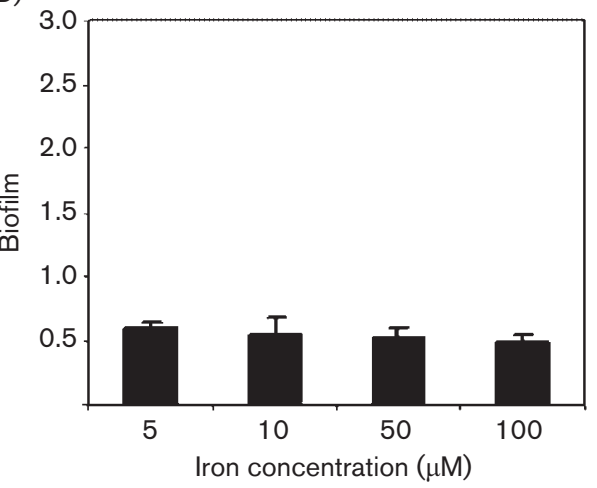

Fig. 1. $P$. aeruginosa $\mathrm{PAO} 1$ was grown for $24 \mathrm{~h}$ in microtitre tray wells containing $\mathrm{AB}$-glucose medium with different concentrations of $\mathrm{FeCl}_{3}$, then culture densities, biofilm amounts and extracellular DNA amounts were determined. (A) Final $\mathrm{OD}_{600}$ of the cultures. (B) Relative amounts of biofilm in the wells determined via a crystal violet staining assay. (C) Relative amounts of extracellular DNA in the cultures determined via a propidium iodide staining assay. (D) Relative amounts of biofilm in the wells after $3 \mathrm{~h}$ of DNase I treatment. Means and standard deviations of eight replicates are shown.

and does not penetrate live bacteria) and measured the level of fluorescence in the cultures after 1 day of growth. The amount of extracellular DNA in $P$. aeruginosa PAO1 microtitre tray cultures was high in medium supplemented with $5 \mu \mathrm{M}$ iron, and decreased with increasing iron concentrations (Fig. 1C). Treatment of 1-day-old $P$. aeruginosa PAO1 biofilms with DNase I led to dispersal of large amounts of the biofilms (Fig. 1D), showing that extracellular DNA plays a critical role in biofilm formation in the experimental setup used.

\section{Iron affects the Pseudomonas quinolone signal (PQS) pathway}

In a previous report, we presented evidence that release of extracellular DNA from $P$. aeruginosa populations was regulated through the $p q s$ quorum-sensing system (AllesenHolm et al., 2006). To investigate whether the observed effect of iron on DNA release from $P$. aeruginosa populations occurs through the PQS system, we initially tested DNA release from different PQS mutants in microtitre tray cultures supplemented with different concentrations of iron. The mutants $p q s A$ and $p q s R$ were found to release less extracellular DNA than the wild-type in an iron-independent manner, whereas a $p q s L$ mutant was found to release more extracellular DNA than the wild-type via a pathway that was suppressed by high iron concentrations (Fig. 2). These results are in agreement with evidence reported by D'Argenio et al. (2002) that the $p q s A$ and $p q s R$ mutants were deficient in PQS production and autolysis, whereas the $p q s L$ mutant overproduced PQS and was hyperautolysing.

To further investigate whether the observed effect of iron on DNA release and biofilm formation might be caused by an effect on the PQS pathway, we constructed a plasmid-based $p q s A:: g f p(A S V)$ transcriptional reporter (as detailed in Methods). The $g f p(A S V)$ gene encodes an unstable version of Gfp (Andersen et al., 1998) and the $p q s A:: g f p(A S V)$ reporter-construct is thus useful for monitoring pqs $A B C D E$ expression. The plasmid with the reporter-construct was transferred to the $P$. aeruginosa wild-type as well as to an isogenic $P$. aeruginosa lasI rhlI quorum-sensing mutant, and $p q s A:: g f p(A S V)$ expression was measured in microtitre tray cultures. The experiments confirmed that the pqsABCDE operon is under $N$-acylhomoserine lactone (AHL) quorumsensing control, as expression of the $p q s A:: g f p(A S V)$ reporter consistently was lower in the lasI rhlI quorum-sensing mutant 


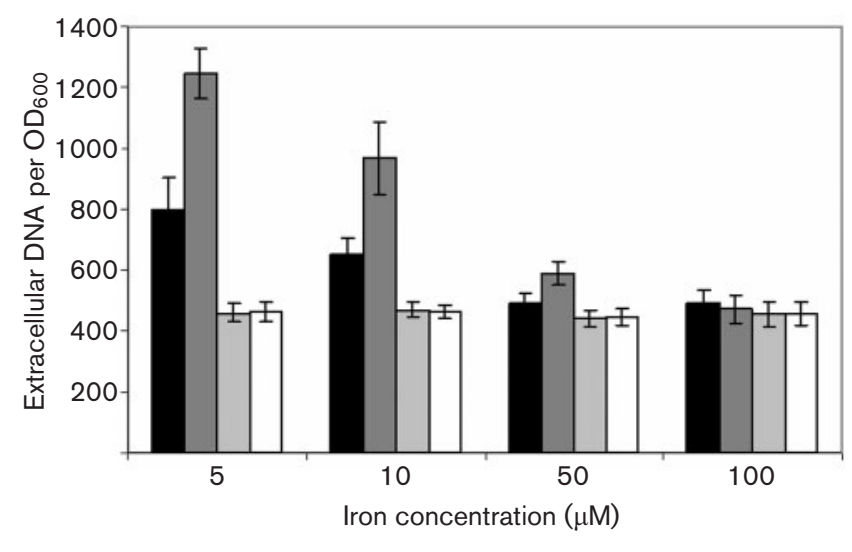

Fig. 2. Relative amounts of extracellular DNA, determined via a propidium iodide staining assay, in microtitre tray cultures of PAO1 wild-type (black bars), pqs $L$ (dark grey bars), pqs A (light grey bars) and pqs $R$ (white bars) grown in AB-glucose medium containing different amounts of $\mathrm{FeCl}_{3}$. Means and standard deviations of eight replicates are shown.

than in the wild-type (Fig. 3A). Transcription of the $p q s A B C D E$ operon in the $P$. aeruginosa wild-type was found to be high in medium supplemented with $5 \mu \mathrm{M}$ iron, and decreased with increasing iron concentrations (Fig. 3A). In the lasI rhlI quorum-sensing mutant there was also an inverse relation between $p q s A:: g f p(A S V)$ expression and the level of iron in the medium (Fig. 3A), suggesting that the effect of iron on $p q s A B C D E$ expression to some extent is independent of the AHL quorum-sensing system. DNA release in the microtitre tray cultures was found to correlate with the expression of the pqsABCDE operon (Fig. 3B). Based on these results, we suggest that iron affects $P$. aeruginosa DNA release and biofilm formation by affecting the PQS signalling pathway.
In order to address whether the observed effects of ferric iron supplementation could be due to induction of an oxidative stress response in the $P$. aeruginosa cells, we investigated microtitre tray cultures supplemented with different concentrations (ranging from 0 to $10 \mathrm{mM}$ ) of $\mathrm{H}_{2} \mathrm{O}_{2}$, and found no reduction in $p q s A:: g f p(A S V)$ expression, DNA release, or biofilm formation (data not shown), indicating that the observed effects of iron are not due to an oxidative stress response.

\section{Iron affects $\boldsymbol{P}$. aeruginosa DNA release and structural biofilm development in flow- chambers}

In order to examine the effect of iron concentration on DNA release in $P$. aeruginosa biofilms, we grew the PAO1 wildtype in flow-chambers irrigated with normal FAB medium (containing $1 \mu \mathrm{M}$ iron) or FAB100 medium (containing $100 \mu \mathrm{M}$ iron). Because of the continuous supply of medium, the amount of iron available to the bacteria will be higher in the flow-chamber setup than in the microtitre tray setup, and we therefore chose to use $\mathrm{FAB}$, containing $1 \mu \mathrm{M}$ iron, as the medium with the lowest iron concentration in the investigations. After 4 days of growth, the extracellular DNA in the biofilms was visualized by staining with the compound DDAO followed by CLSM. In agreement with our previous study (Allesen-Holm et al., 2006), the biofilms grown in FAB medium contained large amounts of extracellular DNA in the part of the biofilm close to the substratum and in the outer region of the stalk portions of the mushroom-shaped multicellular structures (Fig. 4A). In agreement with the quantifications of extracellular DNA and biofilm formation in the microtitre tray assays (described above), the biofilms grown in FAB100 medium contained less extracellular DNA and less biomass than the biofilms grown in normal FAB medium (Fig. 4B). In addition, biofilms grown in FAB100 medium appeared less spatially
(A)

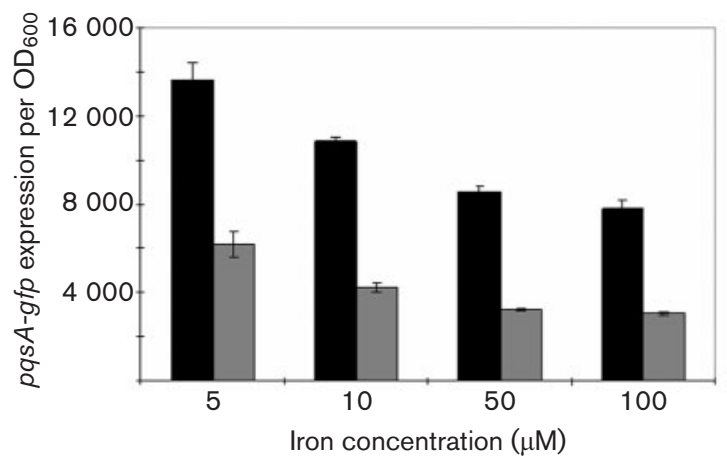

(B)

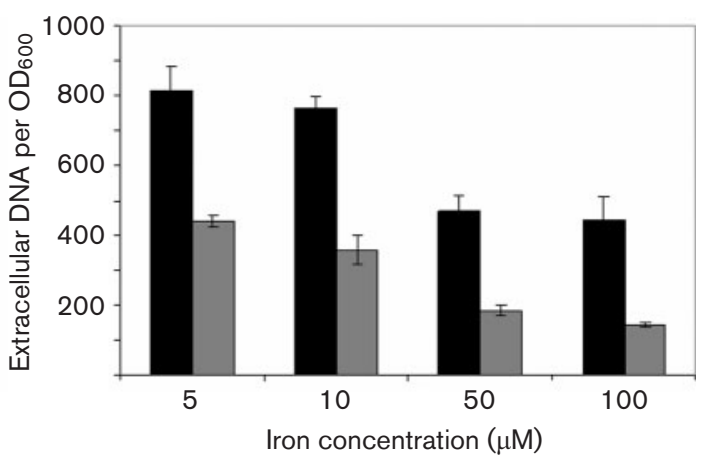

Fig. 3. (A) Expression of a plasmid-borne $p q s A:: g f p(A S V)$ fusion in microtitre tray cultures of the PAO1 wild-type (black bars) and a las/ rhll mutant (grey bars) grown in AB-glucose medium with different concentrations of $\mathrm{FeCl}_{3}$. (B) Relative amounts of extracellular DNA, determined via a propidium iodide staining assay, in microtitre tray cultures of the PAO1 wildtype (black bars) and las/ rhll mutant (grey bars) grown in AB-glucose medium containing different amounts of $\mathrm{FeCl}_{3}$. Means and standard deviations of eight replicates are shown. 

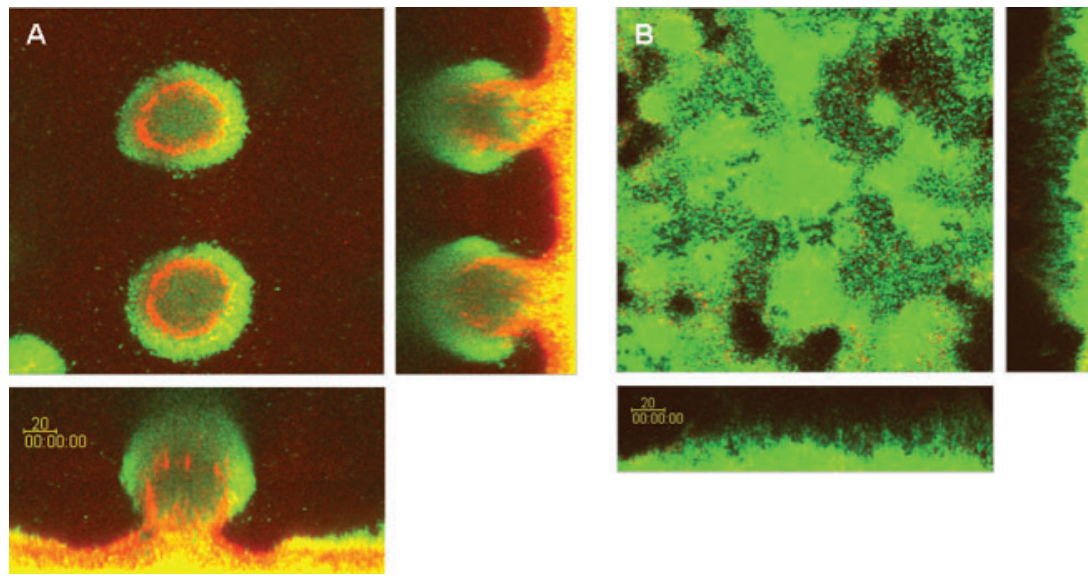

Fig. 4. Four-day-old biofilms of gfp-tagged PAO1 grown in FAB medium (A) or FAB100 medium (B) were stained with DDAO and images visualizing cells (green) and extracellular DNA (appearing red or yellow) were acquired by CLSM. The central pictures show horizontal optical sections, and the flanking pictures show vertical optical sections. Bars, $20 \mu \mathrm{m}$. organized and did not contain the mushroom-shaped multicellular structures typical of the biofilms grown in normal FAB medium (Fig. 4). The difference in biomass and spatial structure between biofilms grown in normal FAB medium and FAB100 medium was confirmed by COMSTAT analysis (Table 1).

\section{Iron affects expression of the pqsABCDE operon in flow-chamber-grown $P$. aeruginosa biofilms}

In order to investigate expression of the pqsABCDE operon in $P$. aeruginosa biofilms, we grew $\mathrm{PAOl}$ containing the $p q s A:: g f p(A S V)$ reporter plasmid in flow-chambers irrigated with $\mathrm{FAB}$ medium or $\mathrm{FAB} 100$ medium. In $\mathrm{FAB}$ medium, expression of the $p q s A:: g f p(A S V)$ fusion was detectable at day 1 (Fig. 5A) and reached the highest expression level at day 2 where it was localized mainly in the outer part of the microcolonies (Fig. 5C). After day 2, expression of the $p q s A:: g f p(A S V)$ fusion decreased, and at day 4 only a weak green signal could be detected in the stalk portion of the mushroom-shaped multicellular structures (Fig. 5E). In FAB100 medium, there was significantly less expression of the $p q s A:: g f p(A S V)$ fusion, and this expression appeared less coordinated in time and space (Fig. 5B, D and F).

\section{Iron affects tolerance to antimicrobial compounds in flow-chamber-grown $\boldsymbol{P}$. aeruginosa biofilms}

Since we had found that the level of iron in the medium may control whether a structured or an unstructured $P$. aeruginosa biofilm is formed, and because tolerance to antimicrobial compounds in biofilms may be related to the spatial structure, we found it of interest to investigate tolerance to the antibiotic ciprofloxacin and to the antimicrobial compound EDTA in P. aeruginosa biofilms grown in FAB medium or FAB100 medium. After 4 days of growth, the biofilms were treated with either ciprofloxacin or EDTA, after which the dead bacteria in the biofilms were visualized by staining with propidium iodide. In 4-day-old $P$. aeruginosa $\mathrm{PAO} 1$ biofilms grown in $\mathrm{FAB}$ medium, ciprofloxacin treatment caused killing of the bacteria located in the outer part of the cap portion of the mushroomshaped structures (Fig. 6C), whereas EDTA treatment caused killing of the cells located within the mushroomshaped structures (Fig. 6E). In 4-day-old $P$. aeruginosa PAO1 biofilms grown in FAB100 medium, treatment with ciprofloxacin or EDTA appeared to kill a large proportion of the bacteria, and there was no special area for the ciprofloxacin- or EDTA-mediated killing (Fig. 6D, F). Propidium iodide staining of biofilms that had not been treated with antibiotic showed that virtually no dead cells

Table 1. Quantification of biofilm architecture

The results are means of datasets obtained from analysis of eight CLSM images acquired at random positions in each of the biofilms. Standard deviations are shown in parentheses.

\begin{tabular}{|c|c|c|c|c|c|c|}
\hline Biofilm medium & $\begin{array}{l}\text { Total biomass } \\
\qquad\left(\mu \mathrm{m}^{3} \mu \mathrm{m}^{-2}\right)\end{array}$ & $\begin{array}{c}\text { Mean thickness } \\
(\mu \mathrm{m})\end{array}$ & $\begin{array}{r}\text { Roughness } \\
\text { coefficient }\end{array}$ & $\begin{array}{l}\text { Surface area } \\
\text { covered }\left(\mu \mathbf{m}^{2}\right)\end{array}$ & $\begin{array}{c}\text { Surface/ } \\
\text { volume }\left(\mu \mathrm{m}^{2} \mu \mathrm{m}^{-3}\right)\end{array}$ & $\begin{array}{l}\text { Max. thickness } \\
(\mu \mathrm{m})\end{array}$ \\
\hline $\begin{array}{l}\text { FAB (containing } \\
\left.1 \mu \mathrm{M} \mathrm{FeCl}_{3}\right)\end{array}$ & $40.06(1.97)$ & $42.82(2.74)$ & $0.39(0.08)$ & $8.71 \times 10^{5}\left(2.72 \times 10^{5}\right)$ & $0.41(0.12)$ & $96.63(3.42)$ \\
\hline $\begin{array}{l}\text { FAB100 (containing } \\
\left.100 \mu \mathrm{M} \mathrm{FeCl}{ }_{3}\right)\end{array}$ & $13.08(4.76)$ & $17.56(5.41)$ & $0.59(0.18)$ & $1.21 \times 10^{6}\left(3.30 \times 10^{5}\right)$ & $1.81(0.36)$ & $59.75(2.12)$ \\
\hline
\end{tabular}



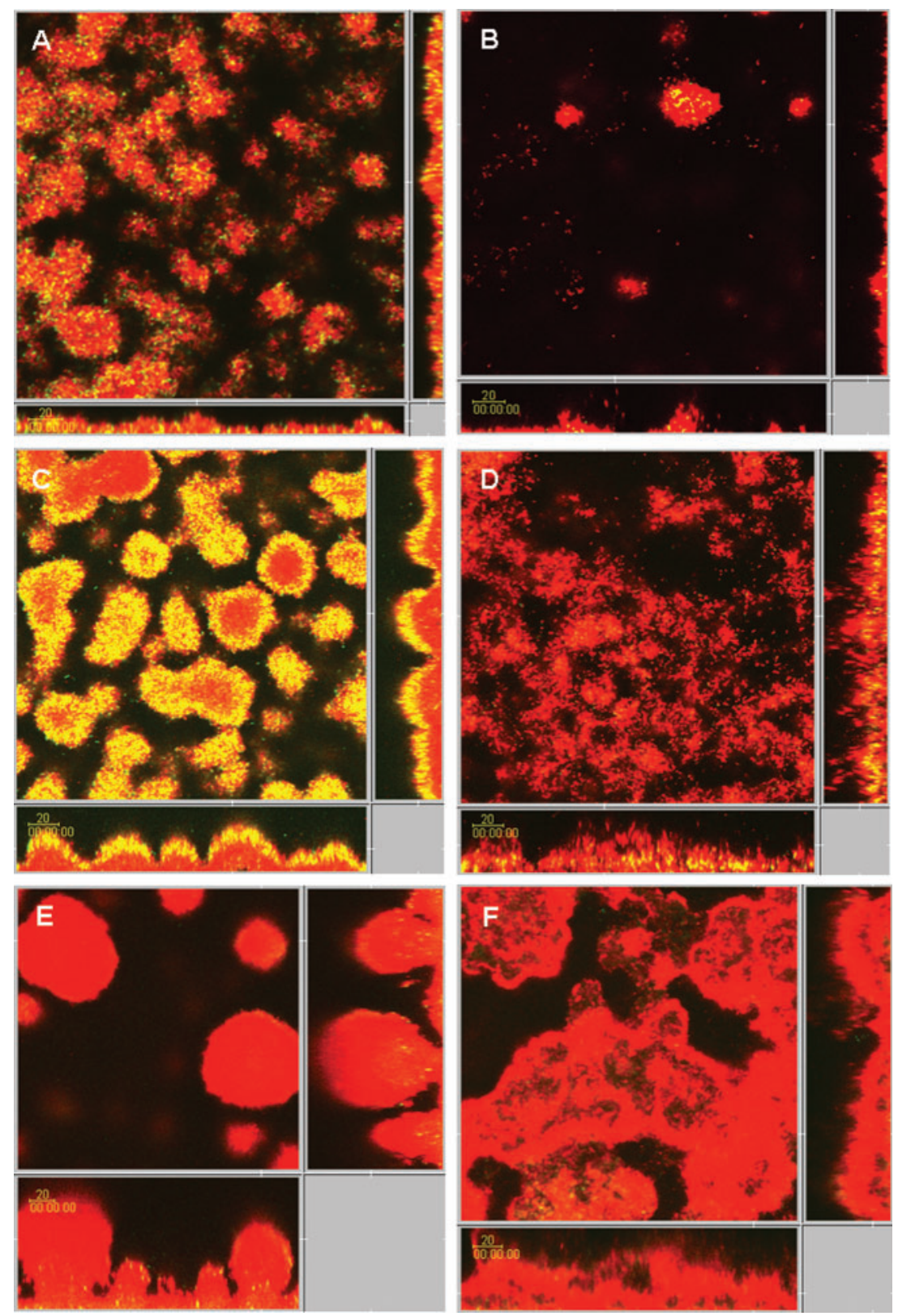

Fig. 5. Biofilms of PAO1 containing a pqsA-gfp(ASV) reporter were grown for 1 $(A, B), 2(C, D)$ and $4(E, F)$ days in flow chambers irrigated with $F A B$ medium (A, C, E) or FAB100 medium (B, D, F). The cells were stained with Syto62, and images visualizing cells (red) and pqsA-gfp(ASV) expression (appearing yellow) were acquired by CLSM. The central pictures show horizontal optical sections, and the flanking pictures show vertical optical sections. Bars, $20 \mu \mathrm{m}$. were present in the biofilms before antibiotic treatment, and that the propidium iodide staining did not visualize extracellular DNA with the setting of the CLSM that was used (Fig. 6A, B). (Propidium iodide does not give as high a signal as DDAO, and with normal settings of the CLSM it will therefore mainly visualize dead cells where the concentration of DNA is high compared to in the biofilm matrix.)

Assessment of survival in the biofilms through recovery of the bacteria from the flow-chambers, after EDTA treatment, ciprofloxacin treatment, or no antimicrobial treatment, followed by plating and c.f.u. counting, confirmed that the structured $P$. aeruginosa biofilms grown in FAB medium displayed higher tolerance to the ciprofloxacin and EDTA treatment than the unstructured $P$. aeruginosa biofilms grown in FAB100 medium. C.f.u. from ciprofloxacintreated biofilms divided by c.f.u. from untreated biofilms was 0.22 (three replicates, SD 0.13) for the biofilms grown in $\mathrm{FAB}$, and 0.02 (three replicates, SD 0.001) for the biofilms grown in FAB100. C.f.u. from EDTA-treated biofilms divided by c.f.u. from untreated biofilms was 0.55 (three replicates, SD 0.095) for the biofilms grown in FAB and 0.21 (three replicates, SD 0.082) for the biofilms grown in FAB100.

\section{DISCUSSION}

A number of recent reports suggest that iron affects biofilm formation by $P$. aeruginosa. Experiments using iron-chelating lactoferrin suggested that a minimum concentration of free 

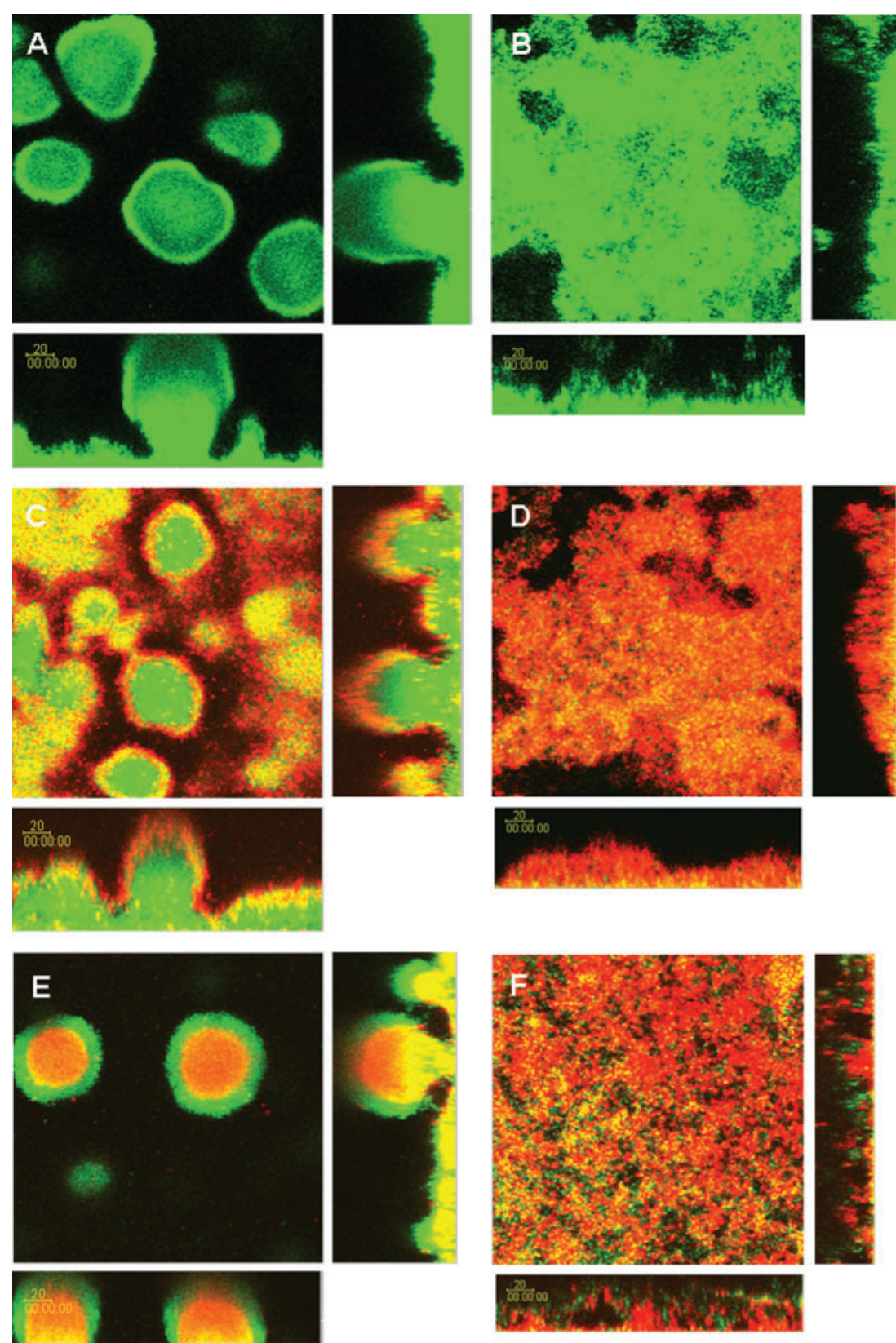

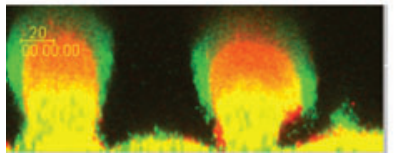

Fig. 6. Four-day-old biofilms of gfp-tagged $P A O 1$ grown in $F A B$ medium $(A, C, E)$ or FAB100 medium ( $B, D, F)$ were either not treated $(A, B)$, treated with ciprofloxacin $(C$, $D)$, or treated with EDTA (E, F), after which they were stained with propidium iodide and images were acquired by CLSM. Live cells appear green and dead cells appear yellow or red. The central pictures show horizontal optical sections, and the flanking pictures show vertical optical sections. Bars, $20 \mu \mathrm{m}$.

iron is necessary for the formation of structured $P$. aeruginosa biofilms in a flow-chamber system (Singh et al., 2002; Banin et al., 2005). On the other hand, high concentrations of iron were shown to suppress $P$. aeruginosa biofilm formation in both microtitre tray and flow-chamber systems (Musk et al., 2005), and low iron concentrations were shown to be required for $P$. aeruginosa biofilm (microcolony) formation in artificial sputum medium (Sriramulu et al., 2005). In agreement with these previous studies, we found in the present study that in our microtitre tray and flow-chamber setups low iron levels promoted biofilm formation while high iron levels suppressed biofilm formation. Experiments involving DNase I treatment of biofilms in microtitre trays (this study) or flow-chambers (Allesen-Holm et al., 2006) suggested that extracellular DNA is an important constituent of the biofilm matrix. Furthermore, evidence was presented that the formation of extracellular DNA in $P$. aeruginosa biofilms is regulated via the PQS quorum-sensing system (Allesen-Holm et al., 2006). In this paper, we present evidence that low iron concentrations promote $P$. aeruginosa biofilm development via up-regulation of the $p q s$ genes and the formation of extracellular DNA, and that high levels of iron suppress $P$. aeruginosa biofilm development via down-regulation of the pqs genes and the formation of extracellular DNA. In agreement with our present and previous work, a study 
comparing different microarray results identified the PQS regulator gene $p q s R$ as being among genes that are induced at low iron levels and also positively controlled by quorum sensing (Cornelis \& Aendekerk, 2004).

Under specific conditions in flow-chamber systems (e.g. the glucose FAB medium used in the present study), $P$. aeruginosa forms heterogeneous biofilms with mushroomshaped multicellular structures via a process that involves formation of microcolonies in the initial phase and subsequent formation of cap-shaped structures on top of the microcolonies in the later phase (e.g. Klausen et al., 2003a). In agreement with the present study, the extracellular DNA in such $P$. aeruginosa biofilms was shown to be present in high concentrations close to the substratum and in the outer part of the microcolonies in the initial phase of biofilm development, and in the outer region of the stalk portion of the mushroom-shaped multicellular structures in the later phase of biofilm development (Allesen-Holm et al., 2006). In the present study we observed that when $P$. aeruginosa formed biofilms in flow-chambers irrigated with FAB medium, expression of a $p q s A:: g f p(A S V)$ fusion was detectable in 1-day-old biofilms and reached the highest expression level at day 2, where it was localized mainly in the outer part of the microcolonies. After day 2, expression of the $p q s A:: g f p(A S V)$ fusion in the biofilms decreased, and at day 4 only a weak green signal could be detected in the stalk portion of the mushroom-shaped multicellular structures. This spatio-temporal expression pattern of pqsABCDE is in accordance with the location of extracellular DNA in $P$. aeruginosa biofilms reported here and by Allesen-Holm et al. (2006). In the medium containing a high iron concentration, there was significantly less expression of the $p q s A:: g f p(A S V)$ fusion, and this expression appeared less coordinated in time and space. In agreement with the quantifications of extracellular DNA and biofilm formation in the microtitre tray assays, and in agreement with the role of the pqs genes in DNA release (Allesen-Holm et al., 2006), the biofilms grown at high iron concentration contained less extracellular DNA and less biomass than the biofilms grown in normal FAB medium. In addition, biofilms grown at high iron concentration appeared less spatially organized and did not develop mushroom-shaped multicellular structures.

The cap-forming and the stalk-forming subpopulation of the mushroom-shaped multicellular structures in $P$. aeruginosa biofilms in many cases display differential tolerance to antimicrobial compounds. For example, the antibiotic tobramycin was shown to kill preferentially bacteria located in the outer part of the cap portion of the mushroom-shaped structures, whereas the antibiotic colistin, the detergent SDS and the chelator EDTA were shown to kill preferentially bacteria in the stalk portion of the mushroom-shaped structures (Bjarnsholt et al., 2005; Banin et al., 2006; Haagensen et al., 2007). Since we had found that the level of iron in the medium may control whether a structured or an unstructured $P$. aeruginosa biofilm is formed, we found it of interest to investigate tolerance to antimicrobial compounds in $P$. aeruginosa biofilms grown in FAB medium or in FAB100 high-iron medium. Ciprofloxacin treatment and EDTA treatment caused killing of cells located in the cap portion and stalk portion, respectively, of the mushroom-shaped structures of 4-day-old $P$. aeruginosa biofilms grown in FAB medium, whereas there was no special area for ciprofloxacin- or EDTA-mediated killing in 4-day-old biofilms grown in high-iron medium. In addition, the structured $P$. aeruginosa biofilms, grown in FAB medium, displayed higher tolerance to the antimicrobial treatment than the unstructured $P$. aeruginosa biofilms, grown in high-iron medium.

The formation of $P$. aeruginosa extracellular DNA is evidently regulated by both quorum sensing and the level of available iron. Other processes that appear to be subject to regulation by the same cues include elastase production and Mn-superoxide dismutase (SOD) production, which were both shown to be quorum-sensing controlled (Hassett et al., 1999; Winson et al., 1995), and also regulated by iron so that they were induced under low-iron conditions but repressed under high-iron conditions (Bjorn et al., 1979; Hassett et al., 1997; Sokol et al., 1982; Bollinger et al., 2001). The fact that iron regulation of these processes to some extent is independent of quorum-sensing regulation makes nutritional override of quorum sensing possible. Other examples of possible nutritional override of quorum sensing include pyocyanin production, which is positively controlled by quorum sensing (Brint \& Ohman, 1995), but can also be induced by phosphate starvation (Hassett et al., 1992). The possibility of nutritional override of quorum sensing should be taken into account when possible strategies for manipulating quorum sensing for controlling bacterial infections are considered.

Evidence was recently presented that biofilm formation by Staphylococcus aureus is induced at low iron concentrations and repressed at high iron concentrations via a mechanism that does not affect expression of polymeric $\mathrm{N}$-acetylglucosamine, which is the major exopolysaccharide implicated in S. aureus biofilm formation (Johnson et al., 2005). Our unpublished results suggest that extracellular DNA plays a role in S. aureus biofilm formation, and that formation of $S$. aureus extracellular DNA occurs at high levels in low-iron medium and at low levels in high-iron medium. It is possible, therefore, that there is a connection between iron concentration, extracellular DNA and biofilm formation also for the Gram-positive organism S. aureus.

The finding that high amounts of iron may inhibit $P$. aeruginosa biofilm formation raises the question whether iron might be used to treat biofilm infections in medical settings. However, because the lack of free iron in the human body in most cases is limiting bacterial growth (Weinberg, 1999), treatment of biofilm-based infections with iron might not be feasible. Nevertheless, the possibility exists that a combination treatment involving antibiotics and iron might be useful. The finding that iron can suppress biofilm 
formation may motivate a search for similar compounds that can suppress biofilm formation but do not stimulate bacterial growth.

\section{ACKNOWLEDGEMENTS}

This work was supported by a grant from the Danish Technical Research Council to T. T.-N.

\section{REFERENCES}

Allesen-Holm, M., Barken, K. B., Yang, L., Klausen, M., Webb, J. S., Kjelleberg, S., Molin, S., Givskov, M. \& Tolker-Nielsen, T. (2006). A characterization of DNA release in Pseudomonas aeruginosa cultures and biofilms. Mol Microbiol 59, 1114-1128.

Andersen, J. B., Sternberg, C., Poulsen, L. K., Bjorn, S. P., Givskov, M. \& Molin, S. (1998). New unstable variants of green fluorescent protein for studies of transient gene expression in bacteria. Appl Environ Microbiol 64, 2240-2246.

Andersen, J. B., Heydorn, A., Hentzer, M., Eberl, L., Geisenberger, O., Christensen, B. B., Molin, S. \& Givskov, M. (2001). gfp-based $N$-acyl homoserine-lactone sensor systems for detection of bacterial communication. Appl Environ Microbiol 67, 575-585.

Baltimore, R. S., Christie, C. D. \& Smith, G. J. (1989). Immunohistopathologic localization of Pseudomonas aeruginosa in lungs from patients with cystic fibrosis. Implications for the pathogenesis of progressive lung deterioration. Am Rev Respir Dis 140, 1650-1661.

Banin, E., Vasil, M. L. \& Greenberg, E. P. (2005). Iron and Pseudomonas aeruginosa biofilm formation. Proc Natl Acad Sci U S A 102, 11076-11081.

Banin, E., Brady, K. M. \& Greenberg, E. P. (2006). Chelator-induced dispersal and killing of Pseudomonas aeruginosa cells in a biofilm. App Environ Microbial 72, 2064-2069.

Bertani, G. (1951). Studies on lysogenesis. I. The mode of phage liberation by lysogenic Escherichia coli. J Bacteriol 62, 293-300.

Bjarnsholt, T., Jensen, P. O., Burmolle, M., Hentzer, M., Haagensen, J. A., Hougen, H. P., Calum, H., Madsen, K. G., Moser, C. \& other authors (2005). Pseudomonas aeruginosa tolerance to tobramycin, hydrogen peroxide and polymorphonuclear leukocytes is quorumsensing dependent. Microbiology 151, 373-383.

Bjorn, M. J., Sokol, P. A. \& Iglewski, B. H. (1979). Influence of iron on yields of extracellular products in Pseudomonas aeruginosa cultures. J Bacteriol 138, 193-200.

Bollinger, N., Hassett, D. J., Iglewski, B. H., Costerton, J. W. \& McDermott, T. R. (2001). Gene expression in Pseudomonas aeruginosa: evidence of iron override effects on quorum sensing and biofilm-specific gene regulation. J Bacteriol 183, 1990-1996.

Brint, J. M. \& Ohman, D. E. (1995). Synthesis of multiple exoproducts in Pseudomonas aeruginosa is under the control of RhlR-RhlI, another set of regulators in strain PAO1 with homology to the autoinducerresponsive LuxR-LuxI family. J Bacteriol 177, 7155-7163.

Cao, H., Krishnan, G., Goumnerov, B., Tsongalis, J., Tompkins, R. \& Rahme, L. G. (2001). A quorum sensing-associated virulence gene of Pseudomonas aeruginosa encodes a LysR-like transcription regulator with a unique self-regulatory mechanism. Proc Natl Acad Sci U S A 98, 14613-14618.

Clark, D. J. \& Maaløe, O. (1967). DNA replication and the division cycle in Escherichia coli. J Mol Biol 23, 99-112.
Cornelis, P. \& Aendekerk, S. (2004). A new regulator linking quorum sensing and iron uptake in Pseudomonas aeruginosa. Microbiology 150, 752-756.

Costerton, J. W., Stewart, P. S. \& Greenberg, E. P. (1999). Bacterial biofilms: a common cause of persistent infections. Science 284, 1318-1322.

D’Argenio, D. A., Calfee, M. W., Rainey, P. B. \& Pesci, E. C. (2002). Autolysis and autoaggregation in Pseudomonas aeruginosa colony morphology mutants. J Bacteriol 184, 6481-6489.

Davey, M. E. \& O'Toole, G. A. (2000). Microbial biofilms: from ecology to molecular genetics. Microbiol Mol Biol Rev 64, 847-867.

Davies, D. G., Parsek, M. R., Pearson, J. P., Iglewski, B. H., Costerton, J. W. \& Greenberg, E. P. (1998). The involvement of cellto-cell signals in the development of a bacterial biofilm. Science 280, 295-298.

Gallagher, L. A., McKnight, S. L., Kuznetsova, M. S., Pesci, E. C. \& Manoil, C. (2002). Functions required for extracellular quinolone signaling by Pseudomonas aeruginosa. J Bacteriol 184, 6472-6480.

Haagensen, J. A., Klausen, M., Ernst, R. K., Miller, S. I., Folkesson, A., Tolker-Nielsen, T. \& Molin, S. (2007). Differentiation and distribution of colistin/SDS tolerant cells in Pseudomonas aeruginosa biofilms. J Bacteriol 189, 28-37.

Haas, B., Kraut, J., Marks, J., Zanker, S. C. \& Castignetti, D. (1991). Siderophore presence in sputa of cystic fibrosis patients. Infect Immun 59, 3997-4000.

Hassett, D. J., Charniga, L., Bean, K., Ohman, D. E. \& Cohen, M. S. (1992). Response of Pseudomonas aeruginosa to pyocyanin: mechanisms of resistance, antioxidant defenses, and demonstration of a manganese-cofactored superoxide dismutase. Infect Immun $\mathbf{6 0}$, 328-336.

Hassett, D. J., Howell, M. L., Sokol, P. A., Vasil, M. L. \& Dean, G. E. (1997). Fumarase $C$ activity is elevated in response to iron deprivation and in mucoid, alginate-producing Pseudomonas aeruginosa: cloning and characterization of fumC and purification of native fumC. J Bacteriol 179, 1442-1451.

Hassett, D. J., Ma, J. F., Elkins, J. G., McDermott, T. R., Ochsner, U. A., West, S. E., Huang, C. T., Fredericks, J., Burnett, S. \& other authors (1999). Quorum sensing in Pseudomonas aeruginosa controls expression of catalase and superoxide dismutase genes and mediates biofilm susceptibility to hydrogen peroxide. $\mathrm{Mol}$ Microbiol 34, 1082-1093.

Hentzer, M., Wu, H., Andersen, J. B., Riedel, K., Rasmussen, T. B., Bagge, N., Kumar, N., Schembri, M. A., Song, Z. \& other authors (2003). Attenuation of Pseudomonas aeruginosa virulence by quorum sensing inhibitors. EMBO J 22, 3803-3815.

Heydorn, A., Nielsen, A. T., Hentzer, M., Sternberg, C., Givskov, M., Ersboll, B. K. \& Molin, S. (2000). Quantification of biofilm structures by the novel computer program COMSTAT. Microbiology 146, 2395-2407.

Høiby, N., Krogh Johansen, H., Moser, C., Song, Z., Ciofu, O. \& Kharazmi, A. (2001). Pseudomonas aeruginosa and the in vitro and in vivo biofilm mode of growth. Microbes Infect 3, 23-35.

Johnson, M., Cockayne, A., Williams, P. H. \& Morrissey, J. A. (2005). Iron-responsive regulation of biofilm formation in Staphylococcus aureus involves Fur-dependent and Fur-independent mechanisms. J Bacteriol 187, 8211-8215.

Klausen, M., Aaes-Jorgensen, A., Molin, S. \& Tolker-Nielsen, T. (2003a). Involvement of bacterial migration in the development of complex multicellular structures in Pseudomonas aeruginosa biofilms. Mol Microbiol 50, 61-68.

Klausen, M., Heydorn, A., Ragas, P., Lambertsen, L., AaesJorgensen, A., Molin, S. \& Tolker-Nielsen, T. (2003b). Biofilm 
formation by Pseudomonas aeruginosa wild type, flagella and type IV pili mutants. Mol Microbiol 48, 1511-1524.

Lam, J., Chan, R., Lam, K. \& Costerton, J. W. (1980). Production of mucoid microcolonies by Pseudomonas aeruginosa within infected lungs in cystic fibrosis. Infect Immun 28, 546-556.

Latifi, A., Winson, M. K., Foglino, M., Bycroft, B. W., Stewart, G. S., Lazdunski, A. \& Williams, P. (1995). Multiple homologues of LuxR and LuxI control expression of virulence determinants and secondary metabolites through quorum sensing in Pseudomonas aeruginosa PAO1. Mol Microbiol 17, 333-343.

Latifi, A., Foglino, M., Tanaka, K., Williams, P. \& Lazdunski, A. (1996). A hierarchical quorum-sensing cascade in Pseudomonas aeruginosa links the transcriptional activators LasR and RhIR (VsmR) to expression of the stationary-phase sigma factor RpoS. Mol Microbiol 21, 1137-1146.

Matsukawa, M. \& Greenberg, E. P. (2004). Putative exopolysaccharide synthesis genes influence Pseudomonas aeruginosa biofilm development. J Bacteriol 186, 4449-4456.

Miller, J. H. (1972). Experiments in Molecular Genetics. Cold Spring Harbor, NY: Cold Spring Harbor Laboratory.

Musk, D. J., Banko, D. A. \& Hergenrother, P. J. (2005). Iron salts perturb biofilm formation and disrupt existing biofilms of Pseudomonas aeruginosa. Chem Biol 12, 789-796.

Nemoto, K., Hirota, K., Murakami, K., Taniguti, K., Murata, H., Viducic, D. \& Miyake, Y. (2003). Effect of Varidase (streptodornase) on biofilm formed by Pseudomonas aeruginosa. Chemotherapy 49, 121-125.

Ochsner, U. A., Wilderman, P. J., Vasil, A. I. \& Vasil, M. L. (2002). GeneChip expression analysis of the iron starvation response in Pseudomonas aeruginosa: identification of novel pyoverdine biosynthesis genes. Mol Microbiol 45, 1277-1287.

O'Toole, G. A. \& Kolter, R. (1998). Flagellar and twitching motility are necessary for Pseudomonas aeruginosa biofilm development. Mol Microbiol 30, 295-304.

Palmer, K. L., Mashburn, L. M., Singh, P. K. \& Whiteley, M. (2005). Cystic fibrosis sputum supports growth and cues key aspects of Pseudomonas aeruginosa physiology. J Bacteriol 187, 5267-5277.

Passador, L., Cook, J. M., Gambello, M. J., Rust, L. \& Iglewski, B. H. (1993). Expression of Pseudomonas aeruginosa virulence genes requires cell-to-cell communication. Science 260, 1127-1130.

Pesci, E. C., Milbank, J. B., Pearson, J. P., McKnight, S., Kende, A. S., Greenberg, E. P. \& Iglewski, B. H. (1999). Quinolone signaling in the cell-to-cell communication system of Pseudomonas aeruginosa. Proc Natl Acad Sci U S A 96, 11229-11234.
Ratledge, C. \& Dover, L. G. (2000). Iron metabolism in pathogenic bacteria. Annu Rev Microbiol 54, 881-941.

Rombel, I. T., McMorran, B. J. \& Lamont, I. L. (1995). Identification of a DNA sequence motif required for expression of iron-regulated genes in pseudomonads. Mol Gen Genet 246, 519-528.

Sambrook, J., Fritsch, E. F. \& Maniatis, T. (1989). Molecular Cloning: a Laboratory Manual, 2nd edn, vol. 1. Cold Spring Harbor, NY: Cold Spring Harbor Laboratory.

Sauer, K., Camper, A. K., Ehrlich, G. D., Costerton, J. W. \& Davies, D. G. (2002). Pseudomonas aeruginosa displays multiple phenotypes during development as a biofilm. J Bacteriol 184, 1140-1154.

Singh, P. K., Parsek, M. R., Greenberg, E. P. \& Welsh, M. J. (2002). A component of innate immunity prevents bacterial biofilm development. Nature 417, 552-555.

Sokol, P. A., Cox, C. D. \& Iglewski, B. H. (1982). Pseudomonas aeruginosa mutants altered in their sensitivity to the effect of iron on toxin A or elastase yields. J Bacteriol 151, 783-787.

Sriramulu, D. D., Lünsdorf, H., Lam, J. S. \& Römling, U. (2005). Microcolony formation: a novel biofilm model of Pseudomonas aeruginosa for the cystic fibrosis lung. J Med Microbiol 54, 667-676.

Sternberg, C. \& Tolker-Nielsen, T. (2005). Growing and analyzing biofilms in flow cells. In Current Protocols in Microbiology, 1B.2.1-1B.2.15. Edited by R. Coico, T. Kowalik, J. Quarles, B. Stevenson \& R. Taylor. New York: Wiley.

Sutherland, I. W. (2001). The biofilm matrix - an immobilized but dynamic microbial environment. Arch Microbiol 9, 222-227.

Weinberg, E. D. (1999). Iron loading and disease surveillance. Emerg Infect Dis 5, 346-352.

Whitchurch, C. B., Tolker-Nielsen, T., Ragas, P. C. \& Mattick, J. S. (2002). Extracellular DNA required for bacterial biofilm formation. Science 295, 1487.

Winson, M. K., Camara, M., Latifi, A., Foglino, M., Chhabra, S. R., Daykin, M., Bally, M., Chapon, V., Salmond, G. P. \& other authors (1995). Multiple $N$-acyl-L-homoserine lactone signal molecules regulate production of virulence determinants and secondary metabolites in Pseudomonas aeruginosa. Proc Natl Acad Sci U S A 92, 9427-9431.

Worlitzsch, D., Tarran, R., Ulrich, M., Schwab, U., Cekici, A., Meyer, K. C., Birrer, P., Bellon, G., Berger, J. \& other authors (2002). Effects of reduced mucus oxygen concentration in airway Pseudomonas infections of cystic fibrosis patients. J Clin Invest 109, 317-325.

Edited by: P. E. Kolenbrander 